\title{
SPLENIC LYMPHANGIOMATOSIS: AN AUTOPSY CASE REPORT
}

\author{
Anamika Thakral' ${ }^{1}$, Meena Daveshwar ${ }^{2}$, Hiral Shah ${ }^{3}$
}

\section{HOW TO CITE THIS ARTICLE:}

Anamika Thakral, Meena Daveshwar, Hiral Shah. "Splenic Lymphangiomatosis: An Autopsy Case Report". Journal of Evolution of Medical and Dental Sciences 2015; Vol. 4, Issue 69, August 27; Page: 12083-12086, DOI: $10.14260 /$ jemds/2015/1741

ABSTRACT: Cystic lymphangiomatosis of the spleen is an extremely rare pathologic entity. It is considered to be the result of a developmental malformation of the lymphatic system and can involve the spleen alone or may be a part of multiorgan disease. It is usually seen in children. We report a case of cystic lymphangiomatosis of the spleen occurring in an adult woman presenting with death due to fall down showing accidental finding of splenomegaly with emphasis on the rarity of the case in adult age and on the problems in the differential diagnosis.

KEYWORDS: Lymphangiomatosis, Spleen, Cysts.

INTRODUCTION: Lymphangiomas are benign lymphatic system malformation of obscure aetiology arise from sequestration of lymphatic tissue that fails to communicate normally with the lymphatic system. It can be either solitary or multiple. It affects soft tissue or paranchymal organs in a diffuse or multifocal fashion. They principally occur in children and rarely manifests after age 20. The most common locations of lymphangiomas are the neck and the axillary region ${ }^{1}$. Cystic lymphagiomatosis of the spleen is a very rare entity and is usually solitary and asymptomatic. We report a case of the woman who died of fall down due to vertigo. There was accidental finding of cystic lymphangiomatosis of spleen with mild spleenomegaly. Even though rare, cystic lymphangiomatosis should be considered in the differential diagnosis of multiple focal lesions of the spleen in any age group.

CASE REPORT: We report an autopsy case report of 33 year woman died because of fall down. The lungs, heart, liver with gall bladder, kidneys, spleen and brain were received. All the organs were unremarkable on gross and microscopic examination except spleen. The spleen was remarkably enlarged weighing $900 \mathrm{gms}$ and measuring $19 \times 12 \times 5 \mathrm{~cm}$ in size. External surface was nodular with creamish white areas. Cut surface showed multiloculated cystic areas containing creamish white gelatinous material, size ranging from 0.5 to $3 \mathrm{~cm}$ in diameter replacing the entire spleenic paranchyma. (Figure.1). Histologically, multiple cystic spaces lined by flat endothelial cells and filled with acidophilic homogenous material were found. (Figure. 2). 


\section{CASE REPORT}

Fig. 1: Cut section of spleen showing cysts of varying sizes ranging from $0.1 \mathrm{~cm}$ to $2.5 \mathrm{cms}$ in diameter filled with gelatinous material.

Fig. 2: H \& E x 400 showing multiple cystic spaces lined by flat endothelial cells and filled with eosinophilic homogenous material.

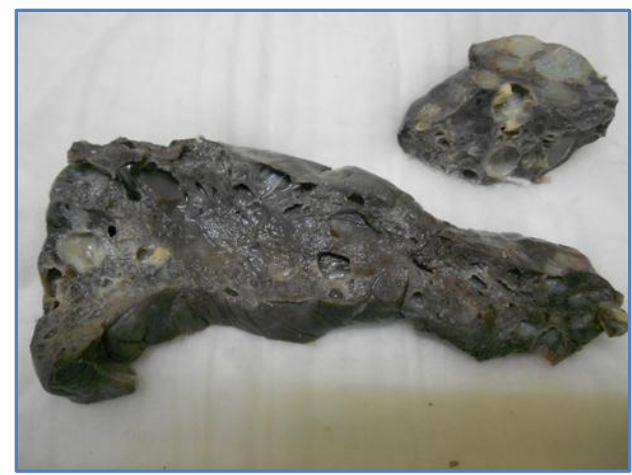

Fig. 1

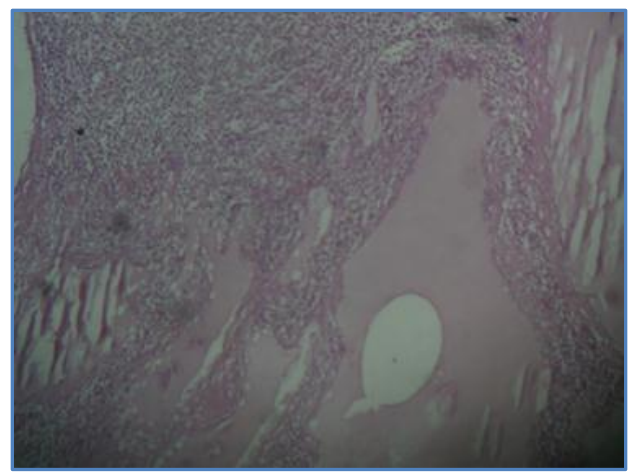

Fig. 2

DISCUSSION: Lymphangiomatosis is a rare, sporadic disorder characterized by cystic lymphangiomas involving multiple organs..$^{2,3}$ The most common sites involved are skin of the face, neck and the axillary region. Involvement of visceral organs like kidney, bones, liver and soft tissues are extremely rare. When multiple organs are involved it is termed as systemic cystic lymphangiomatosis. ${ }^{2}$ In contrast to single lymphangiomas, this form has been reported to be progressive and to have poor prognosis. ${ }^{4}$ The cystic cavities vary in size, from microscopic to several centimetres in diameter. ${ }^{3}$ The formation of cystic lymphangiomas is thought to be due to sequestration or obstruction of lymphatic vessels during embryogenesis. The exact cause is unknown.

Lymphangioma of the spleen is rare and can present as a solitary nodules, multiple nodules or diffuse growth. More often they consist of multiple cysts of varying sizes lined by flat endothelial cells, which contain eosinophilic serous fluid. The septations are made of fibrous connective tissue with rare vessels. ${ }^{5}$

Clinically, the disorder can be classified as systemic, hepatosplenic, or purely splenic or hepatic. Lymphangiomatosis with isolated involvement of the spleen is very rare.6,7,8 It is seen equally among both sexes. It has been described to occur from birth to the seventh decade of life; however, isolated solid organ involvement seems to occur in patients older than 25 years of age. Initial symptoms are usually due to the mass effect caused by the enlarged organs.

At ultrasonography the cysts are hypoechoic with internal debris. The septa are hyperechoic and may show detectable vasculature at colour doppler study. ${ }^{6}$ If the cystic spaces are small and below the resolution of the ultrasound technique, then portions of the mass may appear hyperechoic due to the existence of numerous reflecting interfaces. This may erroneously lead to misdiagnosis.

The differential diagnosis of cystic lymphangiomatosis includes true splenic cysts, mesothelial cysts, echinococcosis and very rarely cystic hamartoma. ${ }^{1}$ True splenic cysts will have definite epithelial lining. They may present with septations but usually the multicystic cavities are larger than in our case. Parasitic cysts most commonly due to Ecchinococcus granulosus may be indistinguishable from any other multicystic mass; therefore they may resemble cystic lymphangiomatosis. 
Serologic test for echinococcosis will be helpful for such condition. Cystic hamartomas are very rare in the spleen, and occur more frequently in the liver in the paediatric age group. ${ }^{7}$ Although cystic lymphangiomatosis of spleen is a benign entity, one case of malignant lymphangioma was also reported. ${ }^{9}$ The malignant degeneration was suggested by the increased proportion of solid areas as in ovarian epithelial neoplasms, papillary excrescences formed by endothelial cells within some of the cystic spaces and solid foci in other regions of the mass.

The normal evolution of splenic lymphangioma is variable. Small lesions are often incidentally detected. Larger lesions may increase in size and cause the compression of other organs or rupture even after minor trauma. Treatment typically consists of fenestration of large cysts or surgical resection of cystic organs. For larger splenic cysts, splenectomy is advantageous, as the risk of rupture is very high. ${ }^{8}$ Splenectomy tends to result in relief of symptoms and in resolution of anaemia and thrombocytopenia in patients with hypersplenism. Cystic lymphangiomatosis of the spleen, even though rare, should be considered in the differential diagnosis of splenomegaly at any age group.

\section{REFERENCES:}

1. Anadol AZ, Oguz M, Bayramoglu H, Edali MN. Cystic lymphangioma of the spleen mimicking hydatid disease. J Clin Gastroenterol 1998; 26:309-311.

2. Seckler SG, Rubin H, Rabinowitz JG. Systemic cystic angiomatosis. Am J Med 1964; 37: 976-986

3. Ramani $P$ and Shah A. Lymphangiomatosis: histologic and immunohistochemical analysis of four cases. Am J Surg Pathol 1993; 17: 329-335.

4. Asch MJ, Cohen AH and Moore T. Hepatic and splenic lymphangiomatosis with skeletal involvement: report of a case and review of the literature. Surgery 1974; 76:334-339.

5. Ito $\mathrm{K}$, Murata $\mathrm{T}$ and Nakanishi $\mathrm{T}$. Cystic lymphangioma of the spleen: MR findings with pathological correlation. Abdom Imaging 1995; 20: 82-84.

6. Komatsuda T, Ishida H, Konno K, Hamashima Y, Naganuma H, Sato M, Ishida J and Masamune 0. Splenic lymphangioma: US and CT diagnosis and clinical manifestations. Abdom Imaging 1999; 24:414-417.

7. Ros PR, Goodman ZD, Ishak KG, Dachman AH, Olmsted WW, Hartman DS and Lichtenstein JE. Mesenchymal hamartoma of the liver: radiologic - pathologic correlation. Radiology 1986; 158: 619-624.

8. Morgenstern L, Bello JM, Fisher BL and Verham RP. The clinical spectrum of lymphangiomas and lymphangiomatosis of the spleen. Am Surg 1992; 58: 599-604.

9. Feigenberg Z, Wysenbeek A and Avidor E. Malignant lymphangioma of the spleen. Isr J Med Sci 1983; 19: 202-204. 


\section{AUTHORS:}

1. Anamika Thakral

2. Meena Daveshwar

3. Hiral Shah

\section{PARTICULARS OF CONTRIBUTORS:}

1. $3^{\text {rd }}$ Year Post Graduate Resident, Department of Pathology, Baroda Medical College, Vadodara.

2. Associate Professor, Department of Pathology, Baroda Medical College, Vadodara.

\section{FINANCIAL OR OTHER} COMPETING INTERESTS: None
3. Tutor, Department of Pathology, Baroda Medical College, Vadodara.

\section{NAME ADDRESS EMAIL ID OF THE} CORRESPONDING AUTHOR:

Dr. Anamika Thakral,

Thakral Hospital,

Kurukshetra Road,

Kaithal, Haryana-136027.

E-mail: anamikathakral@gmail.com

Date of Submission: 10/08/2015.

Date of Peer Review: 11/08/2015.

Date of Acceptance: 24/08/2015.

Date of Publishing: 27/08/2015. 